\title{
Antibody response patterns in chikungunya febrile phase predict protection versus progression to chronic arthritis
}

Kaustuv Nayak, ${ }^{1}$ Vineet Jain, ${ }^{2}$ Manpreet Kaur, ${ }^{1}$ Naushad Khan, ${ }^{3}$ Kamalvishnu Gottimukkala, ${ }^{1}$ Charu Aggarwal, ${ }^{1}$ Rohit Sagar, ${ }^{3}$ Shipra Gupta, ${ }^{3}$ Ramesh Chandra Rai, ${ }^{1}$ Kritika Dixit, ${ }^{1}$ Mohammad Islamuddin, ${ }^{3}$ Wajihul Hasan Khan, ${ }^{3}$ Anil Verma, ${ }^{4}$ Deepti Maheshwari, ${ }^{1}$ Yadya M. Chawla, ${ }^{1}$ Elluri Seetharami Reddy, ${ }^{1}$ Harekrushna Panda, ${ }^{1}$ Pragati Sharma, ${ }^{1}$ Priya Bhatnagar, ${ }^{1}$ Prabhat Singh, ${ }^{1}$ Siva Raghavendhar B, ${ }^{5}$ Ashok Kumar Patel, ${ }^{5}$ Vinod H. Ratageri, ${ }^{6}$ Anmol Chandele, ${ }^{1}$ Pratima Ray, ${ }^{3}$ and Kaja Murali-Krishna ${ }^{1,7,8}$

IICGEB-Emory Vaccine Centre, International Centre for Genetic Engineering and Biotechnology (ICGEB), Aruna Asaf Ali Marg, New Delhi, India. ${ }^{2}$ Department of Medicine, Hamdard Institute of Medical Sciences and Research (HIMSAR), Jamia Hamdard, New Delhi, India. ${ }^{3}$ Department of Biotechnology, School of Chemical \& Life Sciences, Jamia Hamdard, New Delhi, India. ${ }^{4}$ Department of Pediatrics, All India Institute of Medical Sciences, New Delhi, India. ${ }^{5}$ Kusuma School of Biological Sciences, Indian Institute of Technology (IIT), New Delhi, India. ${ }^{6}$ Department of Pediatrics, Karnataka Institute

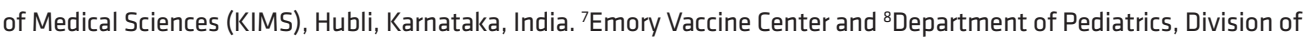
Infectious Diseases, Emory University School of Medicine, Atlanta, Georgia, USA

Chikungunya virus (CHIKV) infection causes acute febrile illness in humans, and some of these individuals develop a debilitating chronic arthritis that can persist for months to years for reasons that remain poorly understood. In this study from India, we characterized antibody response patterns in febrile chikungunya patients and further assessed the association of these initial febrile-phase antibody response patterns with protection versus progression to developing chronic arthritis. We found 5 distinct patterns of the antibody responses in the febrile phase: no CHIKV binding or neutralizing (NT) antibodies but PCR positive, IgM alone with no NT activity, IgM alone with NT activity, IgM and IgG without NT activity, and IgM and IgG with NT activity. A 20-month follow-up showed that appearance of NT activity regardless of antibody isotype or appearance of IgC regardless of NT activity during the initial febrile phase was associated with a robust protection against developing chronic arthritis in the future. These findings, while providing potentially novel insights on correlates of protective immunity against chikungunya-induced chronic arthritis, suggest that qualitative differences in the antibody response patterns that have evolved during the febrile phase can serve as biomarkers that allow prediction of protection or progression to chronic arthritis in the future.

Authorship note: KN and VJ are co-first authors. PR and KMK are co-senior authors.

Conflict of interest: The authors have declared that no conflict of interest exists.

Copyright: (c) 2020, American Society for Clinical Investigation.

Submitted: May 24, 2019

Accepted: March 4, 2020

Published: March 10, 2020

Reference information: JCl Insight. 2020;5(7):e130509.

https://doi.org/10.1172/jci.

insight.130509.

\section{Introduction}

Chikungunya is emerging as an important mosquito-borne arboviral disease of global importance to human health (1-4). Although the virus was originally discovered in Africa in 1952, large outbreaks of chikungunya virus (CHIKV) started appearing from 2005, first in Réunion Island (5), then quickly in India (6-11), followed by a rapid spread to over 40 countries in southeast Asia, the Caribbean, central Europe, and more recently to the Americas (12). Since the major outbreak that occurred in India in 2006, CHIKV has quickly spread throughout the country and now affects millions of people each year (8). Although a vast majority of CHIKV-infected naive individuals develop a febrile illness with joint pain (arthralgia) and/or joint swelling (arthritis), some, but not all, of these affected individuals develop a debilitating chronic arthritis (13-15). The proportion of the affected individuals who develop chronic arthritis is estimated to vary between $10 \%$ and $50 \%$ depending upon the study design and the duration of the follow-up. Although many studies have indicated high viral load (16) or inflammatory mediators (17) are associated with the severity of the disease during the febrile phase, what immune factors that are 
induced during the acute febrile phase predict protection from or progression to chronic arthritis, and its duration, remain poorly understood. Previous studies from our group and other groups indicated that an evolution of antibody responses with neutralizing activity early after onset of chronic disease is important for protection $(18,19)$. What antibody profiles are induced during the febrile phase itself and how they associate with protection versus progression to developing chronic arthritis in the future, especially in a long-term follow-up period, remained less clear.

In this study, we assessed the diversity of the antibody response patterns in chikungunya-confirmed acute febrile patients from India and then evaluated the impact of these febrile antibody response patterns on the downstream outcome of protection versus progression to chronic arthritis in a 20-month follow-up.

\section{Results}

Patients with CHIKV show 5 distinct antibody response patterns during the febrile phase. We recruited 434 patients with chikungunya-suspected symptoms during CHIKV transmission season from 2014 through 2016. The recruitment strategy is outlined in Supplemental Figure 1; supplemental material available online with this article; https://doi.org/10.1172/jci.insight.130509DS1. Of these, 184 patients were confirmed as having CHIKV based on plasma positivity for CHIKV PCR and/or IgM. Among these CHIKV-confirmed cases, 133 were acute febrile cases (fever $<10$ days), and 51 were early or late chronic CHIKV cases (symptoms persisting for 10-40 days after the febrile episode). Clinical and demographic characteristics of these chikungunya-confirmed febrile and chronic patients are shown in Supplemental Table 1.

Analysis of CHIKV-specific IgM and IgG in individual patients revealed 3 broad antibody response patterns that evolved during the CHIKV acute febrile phase (Figure 1A): no detectable IgM or IgG antibody but positive for PCR, positive for IgM alone, or positive for both $\operatorname{IgM}$ and $\operatorname{IgG}$. Among the patients who had induced both IgM and IgG during the febrile phase, $31.9 \%$ had $\operatorname{IgM}>\operatorname{IgG}$ phenotype and $68.1 \%$ had IgG>IgM phenotype. Characterization of neutralizing (NT) activity within these 3 antibody response patterns further expanded them to 5 distinct groups during the febrile phase (Figure 1B). These include group I, no CHIKV binding or NT antibodies but positive for PCR; group II, IgM alone without NT activity; group III, IgM alone with NT activity; group IV, IgM and IgG without NT activity; and group V, IgM and IgG with NT activity. The relative proportion of patients developing each of these 5 distinct antibody patterns in the acute febrile phase is shown in Figure 1C.

Among the isotype-switched febrile cases (groups IV and V), a relatively higher proportion of the NT activity-positive individuals showed an IgG-dominated response (group V, 76\%) compared with the NT activity-negative individuals (group IV, 35\%) (Supplemental Figure 2). These diverse antibody response patterns that were observed during the febrile phase became progressively more uniform toward an IgG-dominated isotype-switched response along with NT activity immediately after the febrile phase during the early chronic phase (Figure $1, \mathrm{~A}-\mathrm{C}$, middle) and late chronic phase (Figure 1, A-C, right).

Taken together, these results show that although patients with chronic CHIKV show a relatively uniform response of IgM, IgG, and NT activity, there is remarkable heterogeneity in the patterns of the antibody responses that evolve during the CHIKV acute febrile phase.

Appearance of NT activity or isotype switching in the febrile phase was associated with low viral loads. We questioned whether these distinct antibody profiles that were observed during the acute febrile phase were simply related to differences in the age of the patient or the days of fever (DOF). Although the mean age of the patient (Figure 2A) and the average DOF (Figure 2B) were strikingly similar among the 5 groups, individuals who had not developed any antibodies but were PCR positive were at marginally earlier DOF (Figure 2B, group I), suggesting that the evolution of these antibody response patterns is likely highly dynamic within the febrile phase. Consistent with this, even among the isotype-switched individuals, IgG was only moderately higher in group V patients (who had developed NT activity) compared with group IV patients (who were lacking NT activity) (Figure 2D, groups IV, V). Analysis of the IgG subtypes revealed that this IgG response, when induced within the febrile phase (i.e., group IV and group V patients), was composed of a mix of IgG1, IgG2, and IgG3 isotypes regardless of the evolution of NT activity (Supplemental Figure 3). The IgM levels, when induced, were strikingly similar regardless of the NT activity or isotype switch status in the acute febrile phase (Figure 2C, groups II, III, IV, V). Although the NT antibody titers were relatively lower in group III (who had IgM alone without NT activity) compared with group V (who had IgM, IgG, and NT activity) in the febrile phase (Figure 2E, group III, V), the appearance of NT activity (groups III and V) as well as the occurrence of 
A
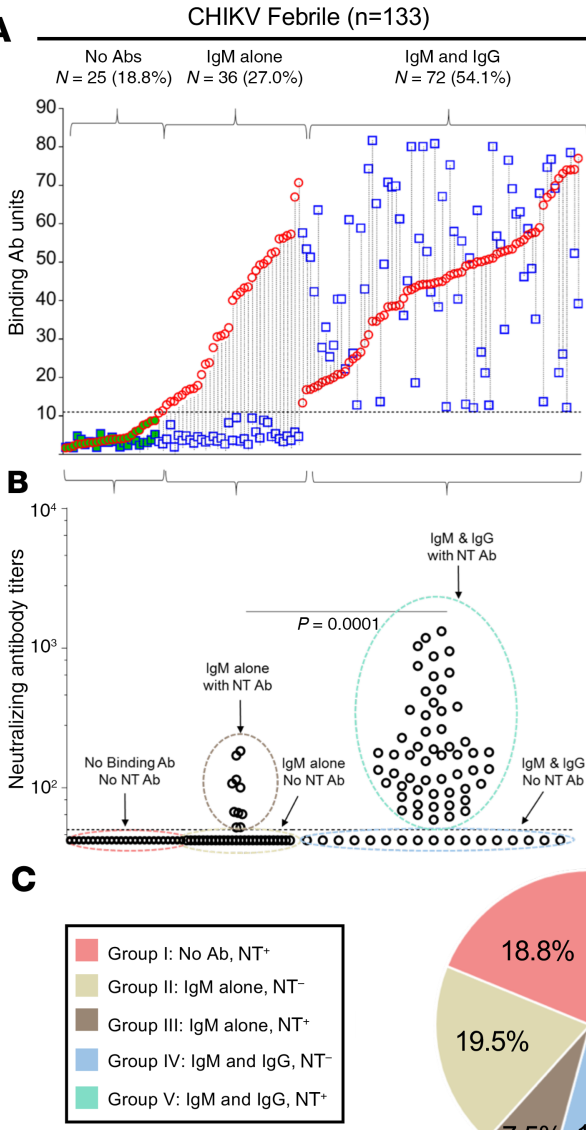

CHIKV Early Chronic $(n=21)$
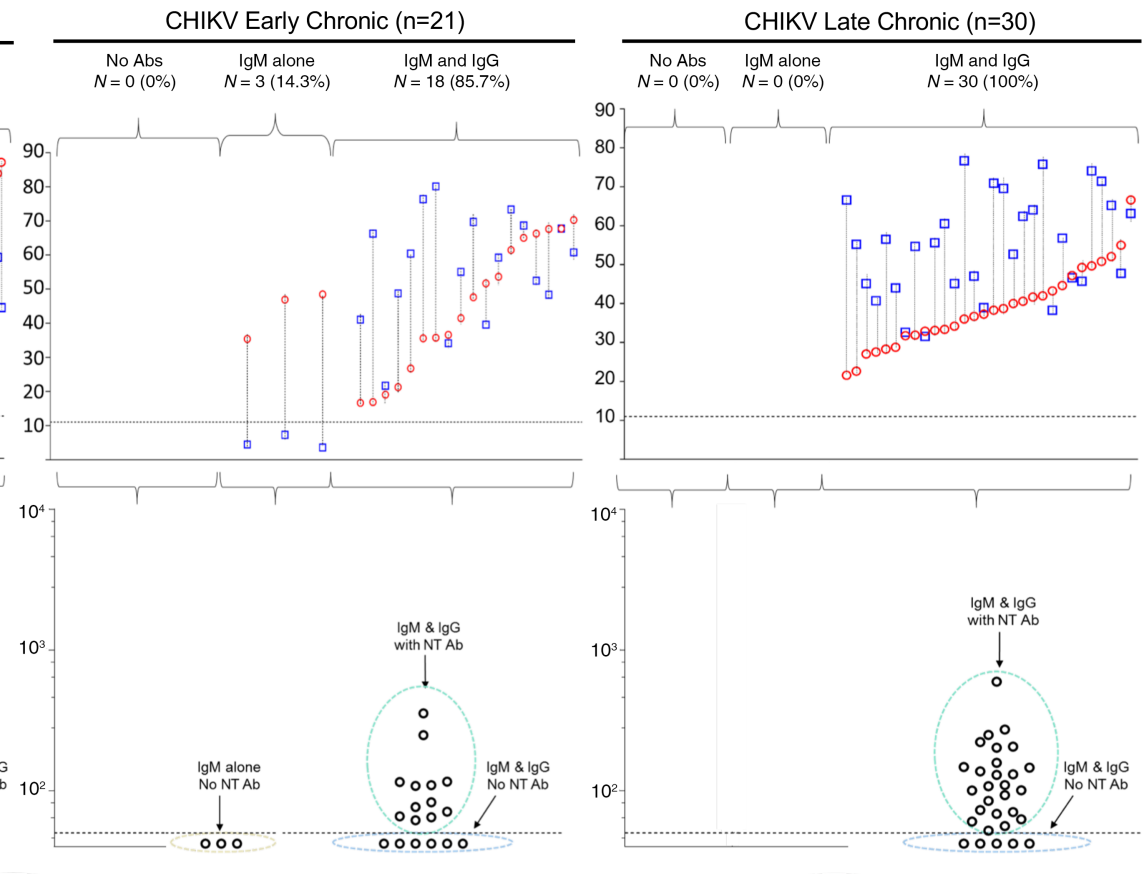
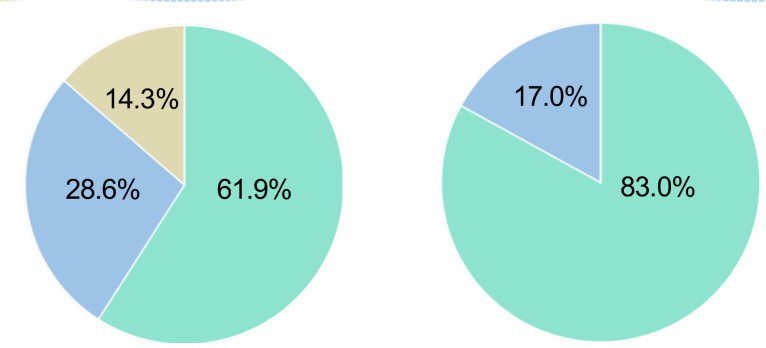

Figure 1. Diversity of the antibody response patterns in chikungunya acute febrile versus chronic patients. (A) Paired analysis of CHIKV-specific plasma IgM (red circles) and IgG (blue squares) values in individual CHIKV-confirmed patients within the no antibodies, IgM-alone, or IgM and IgC antibody response patterns in the acute febrile (left, $n=133$ ), early chronic (middle, $n=21$ ), and late chronic (right, $n=30$ ) phases. Within each antibody response pattern group, the patients are stratified based on increasing IgM values on the $x$ axis. Horizontal dotted line indicates assay cutoff for IgM and IgG. The samples that were also positive for CHIKV PCR are indicated by green-filled symbols. (B) Evaluation of plasma CHIKV NT antibody activity in each of the patient groups that are described in A. Dotted gates were placed to further subgroup the patients based on a combination of IgM, IgG, and NT activity. NT assay limit of detection is indicated by the horizontal dotted line. The NT antibody titers were significantly different between the IgM-alone group and IgM and IgG group in the acute febrile patients. Statistical significance was calculated by unpaired Mann-Whitney $U$ test. (C) Relative proportion of the patients with each of the indicated antibody response patterns shown in B among the CHIKV-confirmed patients in febrile phase (left), early chronic phase (middle), and late chronic phase (right).

isotype switching (groups IV and V) seem associated with better viral control during the febrile phase. This is evidenced by a much lower proportion of the patients in whom virus was recoverable in group III, group IV, and group V patients $(50 \%, 24 \%$, and $22 \%$ respectively) compared with group I or group II patients (100\% and $72 \%$, respectively) (Supplemental Table 2). Consistent with this, the viral load, where recovered, was also found to be lowest in group III, group IV, and group V patients (virus copy numbers $10^{5}$ or less) compared with group I or group II patients (virus copy numbers $10^{8}$ or more) (Figure $2 \mathrm{~F}$ ). Taken together, these results suggested that appearance of NT activity regardless of isotype switch status or occurrence of isotype switching regardless of NT activity was associated with better viral control in the febrile phase.

The NT activity in group III patients was mediated by IgM, whereas the NT activity in group $V$ patients was mediated by either IgG or a combination of $\operatorname{Ig} G$ and $\operatorname{IgM}$. In the plasma of group III febrile patients, we found a tight correlation between the NT titers and IgM levels (Figure 3A). In vitro destruction of IgM led to a decrease in the NT titers of these plasma (Figure 3C), showing that IgM primarily mediates the NT activity in this group. On the other hand, in the plasma of group V patients, although NT antibody titers showed a better correlation with IgG (Figure 3B) than with IgM titers (Figure 3A), in vitro destruction of IgM in these plasma suggested that the NT activity in this group was mediated by $\operatorname{IgG}$ in $52 \%$ of patients 


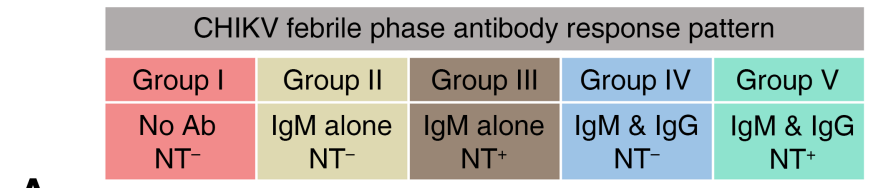

A
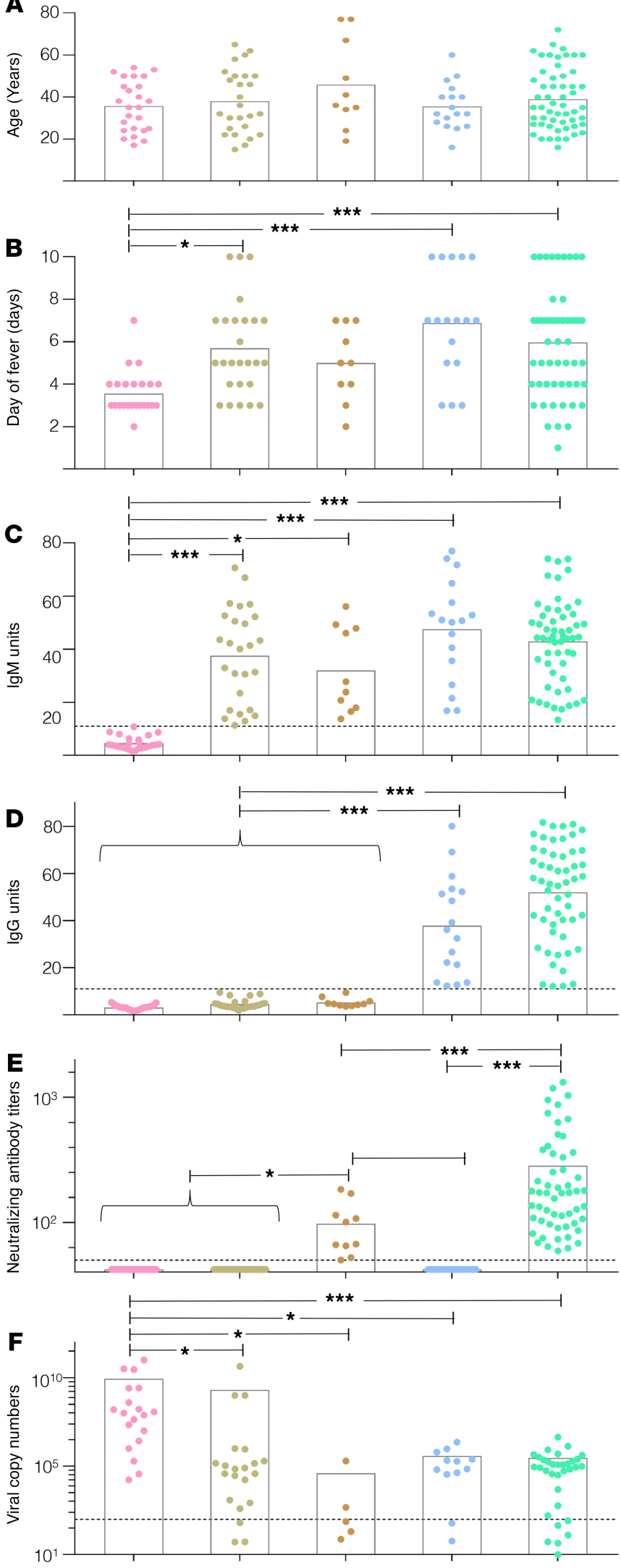

Figure 2. Characterization of age, DOF, antibody response (IgM, IgG, and NT), and viral copy numbers in the acute febrile phase. (A-D) Patients were divided into 5 indicated groups based on their acute febrile antibody response patterns: group I $(n=25)$, group II $(n=26)$, group III ( $n=10)$, group IV $(n=17)$, and group $\vee(n=55)$. (A) Distribution of age. (B) Distribution of the DOF. (C) IgM levels. (D) IgG levels. (E) NT antibody titer values. (F) Viral copy numbers where virus was recovered. Group I $(n=19)$, group II $(n=21)$, group III $(n=5)$, group IV $(n=12)$, and group V $(n=33)$. Mean values for each group are indicated by bars. Comparison between the groups was performed by Kruskal-Wallis 1-way analysis of variance (ANOVA) with Dunn's posttest. ${ }^{* *} P<0.0001$, and ${ }^{*} P<0.05$. Each dot represents an individual patient. Mean values are indicated by bars. Dotted lines indicate the assay cutoff limits for IgM and IgC positivity, for NT titers, or for viral copy numbers.

(Figure 3C, gray lines) and by a combination of $\operatorname{IgM}$ and $\operatorname{IgG}$ in $48 \%$ of these patients (Figure 3C, red lines). Taken together, these results suggested that, among the patients who isotype switched and evolved NT activity, the NT activity was mediated by either IgG or a combination of $\operatorname{IgG}$ and IgM.

Arthritis was observed during the acute febrile phase regardless of the antibody response patterns. We then wondered whether arthritis was associated with any specific antibody response pattern during the acute febrile phase. There was no statistically significant difference in the proportion of patients with arthritis in each of the 5 groups during the acute febrile phase (Figure 4), suggesting that the diverse antibody response patterns induced during the febrile phase have little influence on the frequency of the arthritis cases during the febrile phase.

Appearance of NT activity regardless of antibody isotype or appearance of IgG regardless of NT activity during the febrile phase predicts protection against developing chronic arthritis. To determine whether there is any relation between the antibody response patterns that were induced during the initial febrile phase on future outcome, namely protection versus progression to developing chronic arthritis, we followed up these patients to assess for development of chronic arthritis. A total of 72 patients were successfully assessed for up to 20 months after the febrile phase. Figure 5 shows the percentage of patients with chronic arthritis over the 20-month follow-up period in each of the groups depending on the initial antibody response pattern that they had developed during the initial febrile phase. Development of chronic arthritis was highest in the group I and group II patients. Conversely, the development of chronic arthritis was the least frequent in groups III, IV, and $\mathrm{V}$. Taken together, these observations suggest that appearance of NT activity regardless of antibody isotype or appearance of IgG regardless of NT activity during the acute febrile phase is associated with a better protection against developing chronic arthritis in the future.

\section{Discussion}

Our study provides a comprehensive understanding of the diversity of the humoral response patterns that evolve in chikungunya patients during the acute febrile phase. Additionally, our study provides an understanding on whether these initial febrile antibody response patterns have any correlation with protection versus progression to 


\begin{tabular}{|c|c|c|c|c|}
\hline \multicolumn{7}{|c|}{ CHIKV febrile phase antibody response pattern } \\
\hline Group I & Group II & Group III & Group IV & Group V \\
\hline $\begin{array}{c}\text { No Ab } \\
\mathrm{NT}^{-}\end{array}$ & $\begin{array}{c}\text { IgM alone } \\
\mathrm{NT}^{-}\end{array}$ & $\begin{array}{c}\text { IgM alone } \\
\mathrm{NT}^{+}\end{array}$ & $\begin{array}{c}\text { IgM \& IgG } \\
\mathrm{NT}^{-}\end{array}$ & $\begin{array}{c}\text { IgM \& IgG } \\
\mathrm{NT}^{+}\end{array}$ \\
\hline
\end{tabular}

A

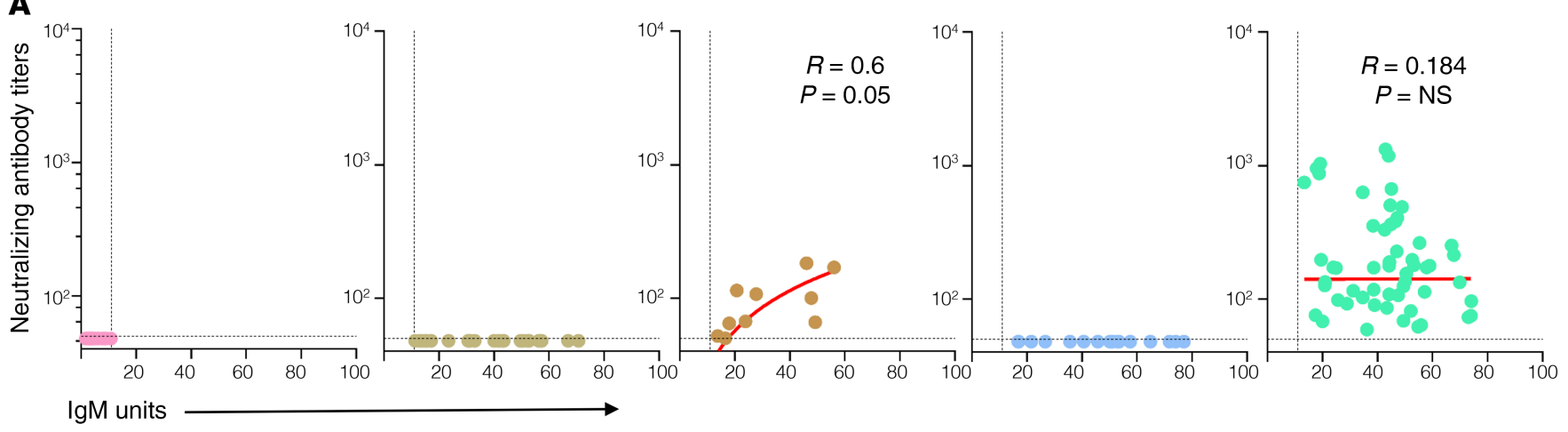

B
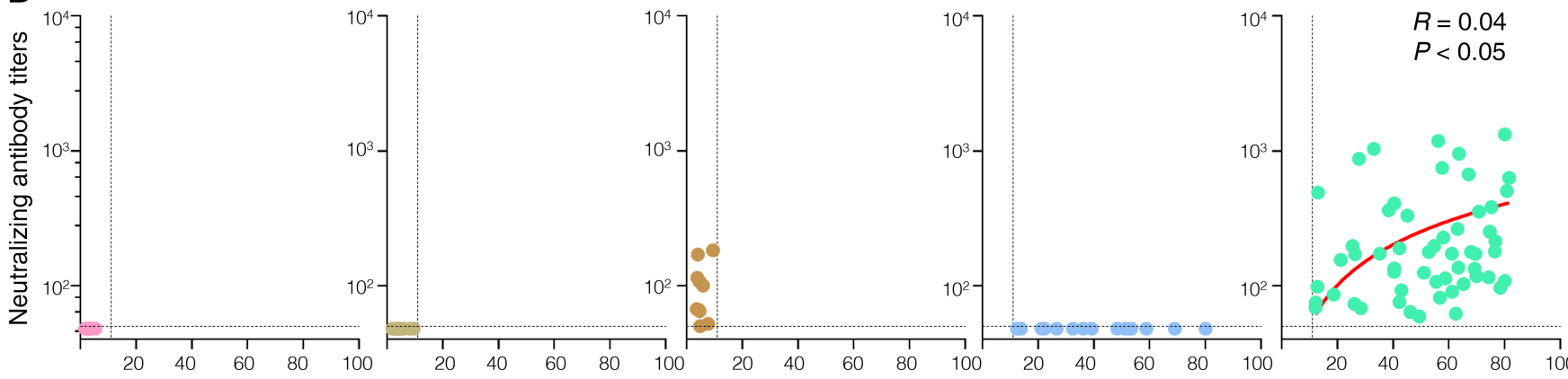

IgG units

C

\begin{tabular}{|c|c|}
\hline \multicolumn{2}{|c|}{ CHIKV febrile patients' antibody response pattern } \\
\hline Group III & Group V \\
\hline IgM alone NT- & IgM \& IgG NT+ \\
\hline
\end{tabular}

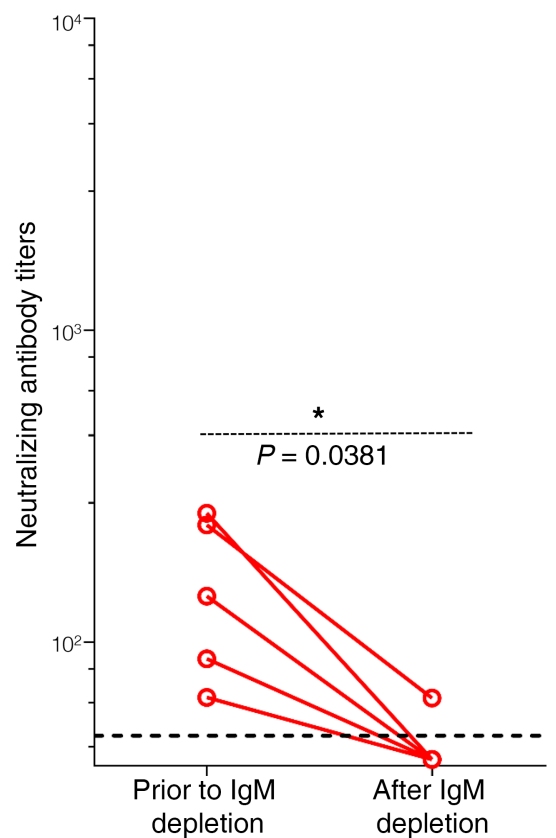

Figure 3. NT activity in the acute febrile phase is contributed by both IgM and IgC. (A) Analysis of correlation between NT antibody titers and IgM levels in plasma of patients from group I ( $n=25)$, group II $(n=26)$, group III $(n=10)$, group IV $(n=17)$, and group $\vee(n=55)$. Dotted line indicates assay cutoff. Spearman's correlation coefficient $r$ was calculated. $P$ values are indicated where significant. (B) Analysis of correlation between NT antibody titers and IgG levels in plasma of patients from group I $(n=25)$, group II $(n=26)$, group III $(n=10)$, group IV $(n=17)$, and group $V(n=55)$. Dotted line indicates assay cutoff. Spearman's correlation coefficient $r$ was calculated, and $P$ values are indicated where significant. (C) NT antibody titers prior to and after IgM depletion. Plasma of group III patients ( $n=$ 5) and group $V$ patients $(n=50)$ was evaluated for NT activity prior to and after IgM destruction. Dotted line indicates assay cutoff. The patients in whom NT titers decreased after IgM depletion are marked in red $(100 \%$ in group III and $48 \%$ in group V). Paired 2-tailed $t$ test was performed to evaluate statistical significance. 


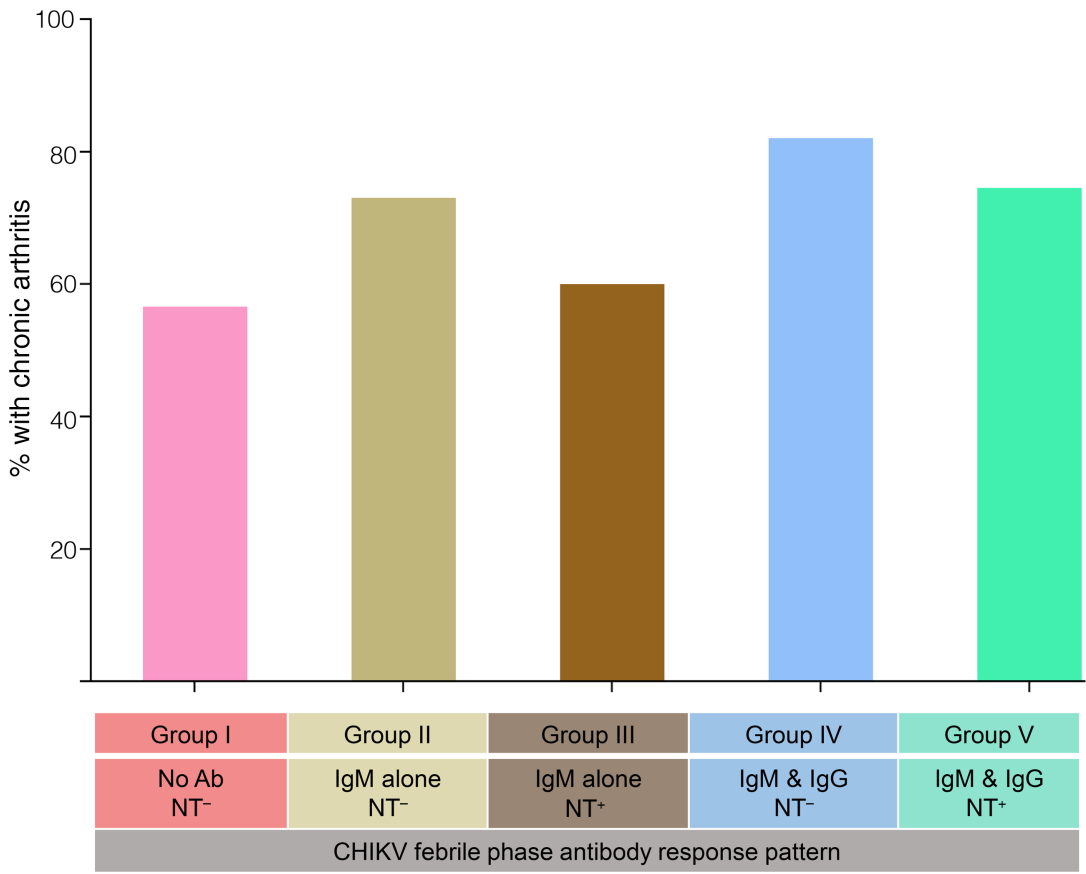

Figure 4. Acute febrile arthritis characteristics of the patients with individual antibody response patterns. Proportion of the arthritis cases in each of the indicated groups during the febrile phase. IgM and IgG NT- $(n=17)$, IgM and IgG NT' $(n=55)$, IgM-only $\mathrm{NT}^{-}(n=26)$, IgM-only $\mathrm{NT}^{+}(n=10)$, and no antibodies $(n=25)$. Statistical significance was calculated by $\chi^{2}$ test. The arthritis case frequency among the groups was not statistically significant between any of the groups $(P>0.05)$.

developing chronic arthritis in the future. We show a remarkable heterogeneity in the antibody response patterns induced during the acute febrile phase. These range from no antibodies, IgM alone without NT activity, IgM alone with NT activity, IgM and IgG without NT activity, and IgM and IgG with NT activity. We show that patients who developed an IgM response with NT activity or an IgM and IgG response with or without NT activity had better viral control in the febrile phase. Using long-term follow-up for over 20 months, we show that the appearance of NT activity regardless of antibody isotype or appearance of IgG regardless of NT activity during the febrile phase is also associated with better protection against developing chronic arthritis in the future. On the other hand, patients who failed to develop NT antibodies or isotype-switched responses during the acute febrile phase (i.e., IgM alone without NT activity) are at the highest risk of developing chronic arthritis in the future. These findings have significance for improving the understanding needed to develop biomarkers of prognostic value as well as to develop and evaluate vaccines, by providing novel insights into correlates of protective immunity against chikungunya-induced chronic arthritis.

Why symptomatic chikungunya patients who are most likely to have primary infections develop such heterogeneous antibody response patterns remains unknown (20). The antibody responses observed during the febrile phase are not static because these responses become more uniform immediately after the febrile phase and in late chronic patients. Additionally, by subsequent sampling of a subset of the patients at 2 years after the febrile phase, we confirmed that both IgG as well as NT activity evolve regardless of the initial febrile response pattern (Supplemental Figure 4). We speculate that these differences arise because of interindividual differences in initial viral inoculum (21), initial viral loads (22), incubation period after viral exposure (23), host genetic factors $(24,25)$, as well as immune responses $(26,27)$ and may contribute to this remarkable heterogeneity in the antibody response patterns that evolve during the acute febrile phase.

An understanding of how the antibody response patterns that were induced during the acute febrile phase tip the balance between protection and pathogenesis is important. A previous study hypothesized that IgM produced during CHIKV infection may lead to pathology by molecular mimicry (28). Our study showing highest chronic arthritis in group II patients (who generated IgM alone without NT activity during the febrile phase) is consistent with this hypothesis. Additionally, our observation that patients who developed an IgM response with NT activity (group III), as well as the patients who developed IgM and IgG response with or without NT activity (groups IV and V), had low viral loads during the acute febrile phase and were highly protected against developing chronic arthritis in the future suggests that these particular antibody response patterns might help tip the balance from pathology to protection, at least in part via better viral control during the febrile phase itself. 


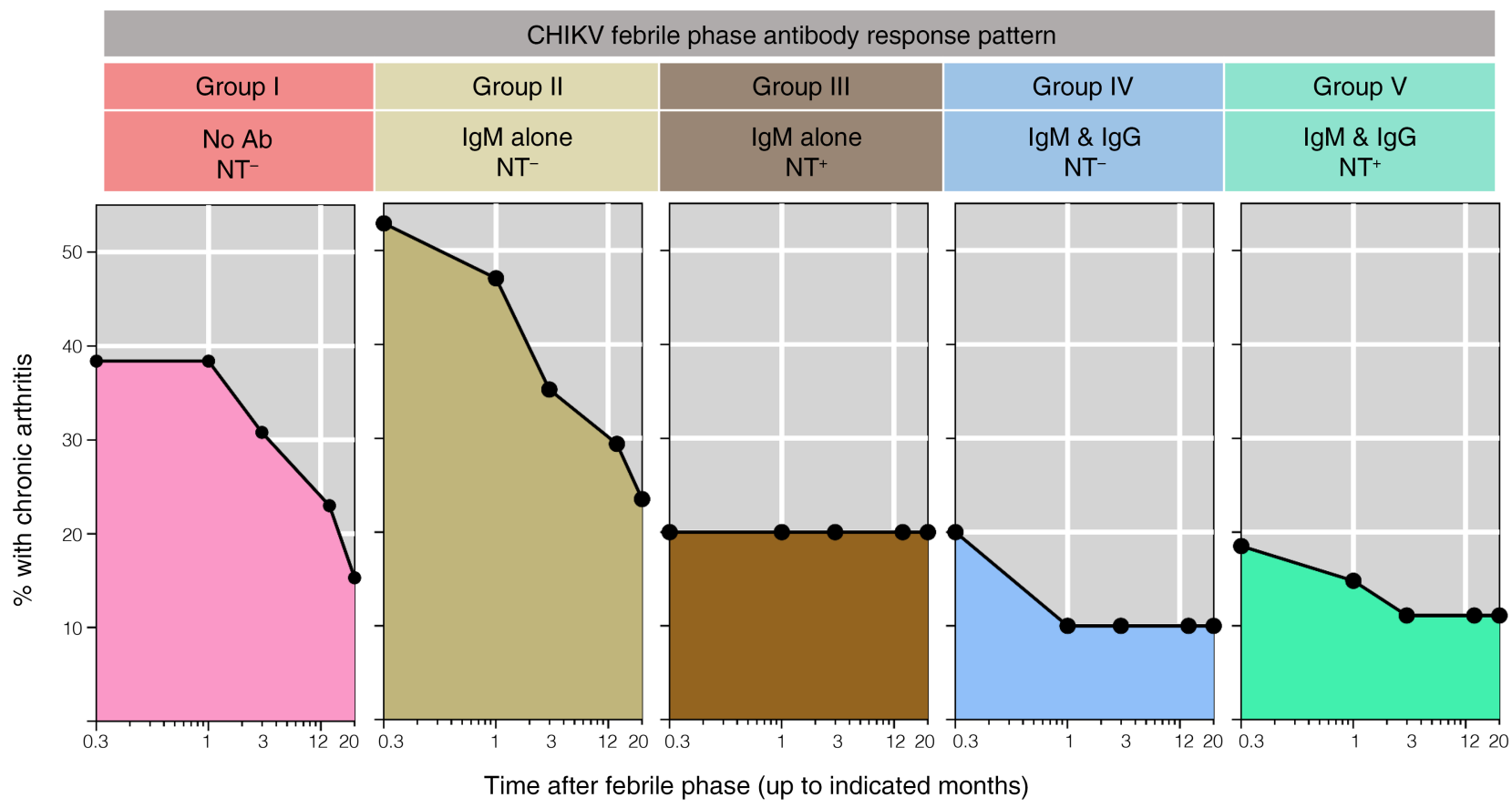

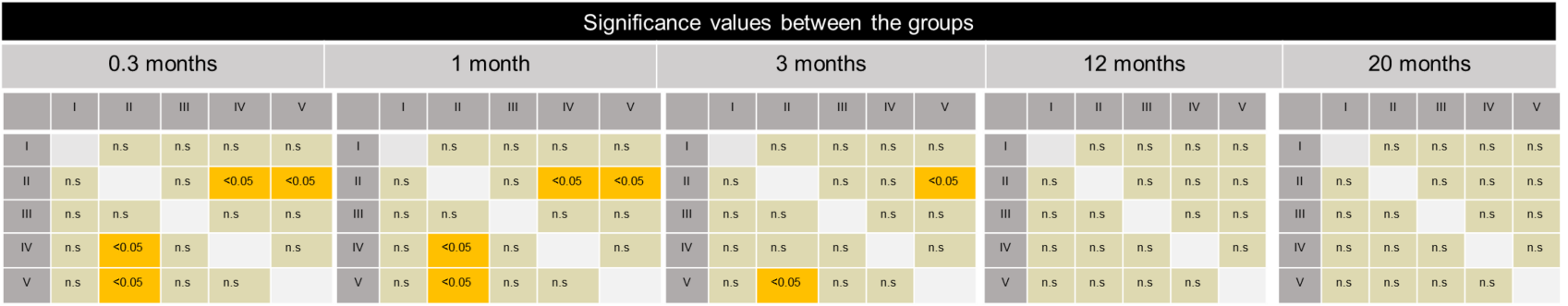

Figure 5. Association of initial febrile antibody response patterns with the downstream fate of protection against developing chronic arthritis. Patients who were followed for more than 20 months as described in Methods were subdivided into the indicated 5 groups based on their initial febrile antibody response pattern. Group I $(n=13)$, group II $(n=17)$, group III $(n=5)$, group IV $(n=10)$, and group V $(n=27)$. The proportion of the patients in each of the group with chronic arthritis is indicated at the indicated times after resolution of fever. Significant values for the proportion of arthritis cases between different groups at 0.3 month, 1 month, 3 months, 12 months, and 20 months are indicated in checkerboards. Significance was calculated using Fisher's exact test of independence. $P$ values less than 0.05 are indicated by yellow color. Where $P$ values were nonsignificant, they are indicated by gray shading.

Although NT activity-mediated viral control is expected, from our studies, it is interesting to note that an early evolution of IgG even without NT activity (group IV) also contributed to protection. Considering that various IgG subtypes can offer protection in an NT-independent way in many viral infections, it is important to know what subtypes are induced in this group. Consistent with our group I pattern, a previous study showed that CHIKV patients who were identified based on PCR positivity largely tend to show no antibody response during the febrile phase (29). Interestingly, this study showed that some, but not all, of these patients who were lacking any antibody response during the febrile phase elicited an isotype-switched IgG3 response that was associated with NT activity soon after the febrile phase. However, it currently remains unclear what IgG subtypes will be induced if an isotype-switched response occurs within the acute febrile phase itself. We have detected both IgG1 and IgG2 as well as IgG3 subtypes in our groups IV (who showed IgG and IgM without NT activity pattern) and V (who showed IgG and IgM with NT activity) during the febrile phase (Supplemental Figure 3). Considering that IgG1 is known to have the longest half-life and to be an efficient mediator of antibody-dependent cellular cytotoxicity, IgG2 is an efficient mediator of phagocytosis whereas both IgG1 and IgG3 robustly activate the complement as well as perform Fc-mediated phagocytosis (30-32). We speculate that these NT-independent effector functions of the IgG subtypes might contribute to the protection observed in group IV patients in our study.

Although the group III, IV, and V patients in our study were highly protected, it is important to note that a small fraction of patients $(\sim 10 \%)$ from these groups continued to have chronic arthritis even after 
a year past the acute episode. This suggests that even though development of a mature isotype-switched antibody response with or without NT activity or IgM response with NT activity during the acute febrile period is a strong correlate of protection, a minor fraction of the individuals are likely to escape protection despite developing these protective antibody response patterns during the febrile phase. Correlation analysis between the NT activity and IgM or IgG magnitude between the individuals who were protected versus those who developed chronic arthritis from groups III, IV, and V showed there were trends, although statistically insignificant, suggesting relatively lower NT activity or IgG in group III, IV, and V patients who were not protected (Supplemental Figure 5, A-C). Whether the group III, IV, and V individuals who escaped the protection differed in other components of innate or adaptive responses, such as innate/inflammatory mediators or $\mathrm{T}$ and $\mathrm{B}$ cell responses, during the febrile phase remains to be addressed.

Interestingly, our results suggest that, in addition to IgG with or without NT activity, the IgM-mediated NT activity may have a beneficial effect in vivo. However, there are 2 limitations in our study. First, all IgG-producing patients ( $\sim 50 \%$ of the febrile patient population) invariably had IgM. Hence, it is difficult to decipher the relative in vivo role of each of these isotypes in protection against developing chronic arthritis. Second, although the "IgM alone with NT activity group" provides a unique opportunity to understand the in vivo role of $\operatorname{IgM}$ in protection against developing arthritis, this group represented only a small fraction (7.5\%) of the total chikungunya febrile patient population that was studied. As a consequence, we could follow up only a small number of the patients from this "IgM alone with NT activity group." Studies are needed, perhaps by recruiting large numbers of patients to identify a sufficiently large set with this IgMassociated NT activity alone, to further solidify this finding.

In summary, our findings suggest that individuals who induce a mature antibody response as evidenced by IgG isotype switching, regardless of the NT activity, or individuals who induce NT activity, regardless of isotype switching, within the acute febrile phase are less prone to chronic arthritis.

\section{Methods}

Patient recruitment. Patients were recruited at 2 sites: HIMSAR, Jamia Hamdard, New Delhi, India; and KIMS, Hubli, Karnataka, India. New Delhi $\left(28.6139^{\circ} \mathrm{N}, 77.2090^{\circ} \mathrm{E}\right)$ is located in the northern part of India, and Hubli $\left(15.3647^{\circ} \mathrm{N}, 75.1240^{\circ} \mathrm{E}\right)$ is located in southern part of India. Both areas have remained highly affected by chikungunya for the past 10 years. Both hospitals have excellent medical care, diagnostics, and accessibility to medical records as per the Government of India standards. Both primary and tertiary care patients visit these hospitals.

Chikungunya-suspected male and female patients aged between 15 and 77 years were recruited in the years 2013-2016 during the chikungunya transmission season, which typically ranges from July through November. The inclusion criteria followed for recruitment included clinical presentation consistent with chikungunya infection, which include abrupt onset of fever within the past 40 days and/or symptoms such as myalgia, rash, fever, body pains, and joint swelling. Pregnant women, patients who were positive for malaria or common respiratory infections such as active tuberculosis, or patients having preexisting arthritis for more than 40 days before the onset of symptoms were excluded. Baseline clinical parameters for disease activity, including joint pain (arthralgia), joint swelling (arthritis), rash, cough, restlessness, myalgia, stomach cramps, and bleeding, were recorded. The most commonly affected joints were wrists, metacarpophalangeal joints, proximal interphalangeal joints, elbows, shoulders, knees, ankles, and metatarsophalangeal joints. A blood specimen drawn at the time of initial clinical evaluation was sent for research studies that run a battery of tests to confirm chikungunya infection or to exclude other febrile infections. Only those patients whose samples were positive for CHIKV PCR and/or IgM and negative for dengue NS1 and/or IgM and who were manifesting acute febrile illness for no more than 10 days were considered acute febrile CHIKV-confirmed patients.

The recruitment criteria are shown in Supplemental Figure 1, and the characteristics of the CHIKVconfirmed patients are shown in Supplemental Table 1. CHIKV-confirmed acute febrile patients were followed up telephonically over the next 2 years. The questionnaire was administered and recorded by clinical care staff, blinded to the serological status, and made available to the research team without personally identifiable information, only after study completion to ensure the best possible double-blinded analysis and anonymity. The questionnaire was composed of closed questions addressing the course of chronic arthritis manifestations and relapse in condition with/without fever to assess the clinical outcome. The status of joint swelling and its resolution was inquired in explicit detail with regard to joint swelling with 
pain and difficulty during walking, bending, squatting, and other day-to-day activities. Data were collected on disease status at 4 time points to a maximum of 20 months in 72 patients.

Serology tests. CHIKV-specific IgM and IgG were detected using chikungunya IgM capture ELISA kits (Abcam ab177848) and chikungunya IgG capture ELISA kit (Abcam ab177835) following the manufacturer's recommendations. Samples were screened for dengue-specific IgM and IgG levels using Panbio dengue IgM Capture ELISA (Panbio 01PE20) and Panbio dengue IgG capture ELISA (Panbio 01PE10) as per the manufacturer's instructions. Samples were screened for dengue NS1 antigen using dengue day-1 test kit (J Mitra \& Co IR028050) as per the manufacturer's instructions and elaborated in our previous studies $(33,34)$.

In-house ELISA for IgG subtypes. IgG subtypes were characterized as described previously (29). CHIKV-coated ELISA plates were incubated with 1:25 diluted plasma followed by washing and incubation with mouse anti-human IgG1 Fc-HRP (Southern Biotech 9054-05), mouse anti-human IgG2 Fc-HRP (Southern Biotech 9060-05), mouse anti-human IgG3 hinge-HRP (Southern Biotech 921005), and mouse anti-human IgG4 Fc-HRP (Southern Biotech 9200-05) at 1:1000 dilution. ELISA color reaction was developed using TMB substrate (Mabtech 3652-F10), and optical density was measured in an ELISA reader at $490 \mathrm{~nm}$ wavelength.

CHIKV NT antibody assays. NT activity of plasma samples was evaluated using PRNT assays as described previously (18). Vero cells (ATCC, CCL-81) were plated on 96-well plates at a concentration of 30,000 cells per well. After overnight culture, the cells were infected with 50 plaque-forming units of CHIKV per well that was preincubated for 1 hour with graded 2-fold dilutions of the heat-inactivated plasma (ranging from 1:50 to 1:3200). After incubation, the cell layer was overlaid with $2 \%$ carboxymethyl cellulose in OptiMEM (Invitrogen, Thermo Fisher Scientific, 31985070) with 2\% FBS, amphotericin B, and ciprofloxacin and incubated for an additional 36 hours. After this, the cell layer was stained using $0.25 \%$ crystal violet in 30\% methanol and the plaques were counted. The NT titer was expressed as the plasma dilution that reduced infectivity by 50\% using nonlinear regression fitting in GraphPad Prism 7.0. Samples that failed to give 50\% plaque reduction at 1:50 dilution were assigned as below the cutoff.

IgM depletion assays. To assess whether the NT activity was associated with IgM, plasma samples from "IgM alone with NT activity" patients were depleted of their IgM activity prior to NT assays as described previously (35). Briefly, heat-inactivated plasma was treated with $0.1 \mathrm{M}$ dithiothreitol (Life Technologies $\mathrm{R} 0861$ ) to a final concentration of $2.5 \mathrm{mM}$ to inactivate $\mathrm{IgM}$, followed by confirmation of loss of $\operatorname{IgM}$ functionality. This plasma was then assessed for its NT activity as described above.

Virological assays. For viral recovery assays, $50 \mu \mathrm{L}$ of patient plasma was diluted with $450 \mu \mathrm{L}$ of DMEM (Invitrogen, Thermo Fisher Scientific, 11965-092) and inoculated onto C6/36 (ATCC CRL-1660) cells in 6-well tissue culture plates. Viral RNA was extracted from culture supernatant using viral RNA extraction kit (QIAGEN 52906) and reverse-transcribed into cDNA using RevertAid First Strand cDNA Synthesis Kit (Thermo Fisher Scientific K1632). PCR was performed by a semiquantitative method using CHIKV/E1-specific primers (CHIK/E1-S 5'-TACCCATTCATGTGGGGC-3', CHIK/E1-C 5'-GCCTTTGTACACCACGATT-3, MilliporeSigma), and the products were analyzed on a $2 \%$ agarose gel. Viral copy numbers were analyzed by real-time PCR as reported earlier (36). For plasma PCR, RNA was extracted from sera using QIAamp Viral RNA Mini Kit (QIAGEN) as per the manufacturer's instructions. PCR was performed as described previously $(36,37)$. Briefly, 3 sets of primers targeting the E1 gene (930 bp, 294 bp, and $200 \mathrm{bp}$ ) were used to confirm CHIKV positivity.

Statistics. Statistical analyses were performed using GraphPad Prism 7.0. Multiple groups were compared using 1-way ANOVA followed by Dunn's posttest. Correlations between 2 groups were calculated by Spearman's correlation coefficient $r$. The unpaired analysis was done using Mann-Whitney $U$ test. Paired groups were compared by 2-tailed $t$ test. Significance between proportions was calculated using Fisher's test. $P \leq 0.05$ was considered significant.

Study approval. The study was approved by the institutional ethical committees (KIMS, HIMSAR, ICGEB), and the participants gave written informed consent.

\section{Author contributions}

KN performed experiments and manuscript preparation; AC, PR, and KMK performed study design, interpretation, and manuscript preparation; VJ and VHR performed patient recruitment and follow-up; and MK, NK, KG, RCR, KD, RS, SG, MI, WHK, AV, DM, CA, YMC, ESR, HP, P Sharma, PB, P Singh, $\mathrm{SRB}$, and $\mathrm{AKP}$ performed data acquisition, maintenance, or analysis. 


\section{Acknowledgments}

This work was supported by Department of Biotechnology, Government of India, and National Institutes of Health Indo-U.S. Vaccine Action Program BT/MB/Indo-US/VAP/06/2013; National Institutes of Health International Collaborations in Infectious Disease Research 1U01AI115651; and the Department of Science and Technology EMR/2016/002475. We thank Rafi Ahmed, Emory Vaccine Center, for his scientific advice. We also thank Satendra Singh and Ajay Singh for their technical help.

Address correspondence to: Kaja Murali-Krishna, Department of Pediatrics, Division of Infectious Diseases, Emory University School of Medicine, 201 Dowman Drive, Atlanta, Georgia 30322 USA. Phone: 404.858.7458; Email: murali.kaja@emory.edu.

1. Silva LA, Dermody TS. Chikungunya virus: epidemiology, replication, disease mechanisms, and prospective intervention strategies. J Clin Invest. 2017;127(3):737-749.

2. Staples JE, Breiman RF, Powers AM. Chikungunya fever: an epidemiological review of a re-emerging infectious disease. Clin Infect Dis. 2009;49(6):942-948.

3. Tetro JA. From hidden outbreaks to epidemic emergencies: the threat associated with neglecting emerging pathogens. Microbes Infect. 2019;21(1):4-9.

4. Vairo F, Haider N, Kock R, Ntoumi F, Ippolito G, Zumla A. Chikungunya: epidemiology, pathogenesis, clinical features, management, and prevention. Infect Dis Clin North Am. 2019;33(4):1003-1025.

5. Paquet C, et al. Chikungunya outbreak in Reunion: epidemiology and surveillance, 2005 to early January 2006. Euro Surveill. 2006;11(2):E060202.3.

6. Kalantri SP, Joshi R, Riley LW. Chikungunya epidemic: an Indian perspective. Natl Med J India. 2006;19(6):315-322.

7. Parashar D, Amdekar S, More A, Patil P, More R, Babu VR. Chikungunya fever outbreak in Guntur, Andhra Pradesh, India. Indian J Med Res. 2015;142(suppl):S111-S1115.

8. Cecilia D. Current status of dengue and chikungunya in India. WHO South East Asia J Public Health. 2014;3(1):22-26.

9. Dinkar A, Singh J, Prakash P, Das A, Nath G. Hidden burden of chikungunya in North India; a prospective study in a tertiary care centre. J Infect Public Health. 2018;11(4):586-591.

10. Lahariya C, Pradhan SK. Emergence of chikungunya virus in Indian subcontinent after 32 years: a review. J Vector Borne Dis. 2006;43(4):151-160

11. Muniaraj M. Fading chikungunya fever from India: beginning of the end of another episode? Indian J Med Res. 2014;139(3):468-470.

12. Centers for Disease Control and Prevention, National Center for Emerging and Zoonotic Infectious Diseases, Division of Vector-Borne Diseases. Chikungunya virus in the United States. Chikungunya Virus website. https://www.cdc.gov/chikungunya/ geo/united-states.html. Accessed March 9, 2020.

13. Daniels NA, Gouveia S, Null D, Gildengorin GL, Winston CA. Acceptance of pneumococcal vaccine under standing orders by race and ethnicity. J Natl Med Assoc. 2006;98(7):1089-1094.

14. Sutaria RB, Amaral JK, Schoen RT. Emergence and treatment of chikungunya arthritis. Curr Opin Rheumatol. 2018;30(3):256-263.

15. Zaid A, Gerardin P, Taylor A, Mostafavi H, Malvy D, Mahalingam S. Chikungunya arthritis: implications of acute and chronic inflammation mechanisms on disease management. Arthritis Rheumatol. 2018;70(4):484-495.

16. Thiberville SD, et al. Chikungunya fever: epidemiology, clinical syndrome, pathogenesis and therapy. Antiviral Res. 2013;99(3):345-370.

17. Kelvin AA, et al. Inflammatory cytokine expression is associated with chikungunya virus resolution and symptom severity. PLoS Negl Trop Dis. 2011;5(8):e1279.

18. Jain J, et al. Clinical, serological, and virological analysis of 572 chikungunya patients from 2010 to 2013 in India. Clin Infect Dis. 2017;65(1):133-140.

19. Kam YW, et al. Longitudinal analysis of the human antibody response to Chikungunya virus infection: implications for serodiagnosis and vaccine development. J Virol. 2012;86(23):13005-13015.

20. Yoon IK, et al. High rate of subclinical chikungunya virus infection and association of neutralizing antibody with protection in a prospective cohort in the Philippines. PLoS Negl Trop Dis. 2015;9(5):e0003764.

21. Welten SPM, Redeker A, Toes REM, Arens R. Viral persistence induces antibody inflation without altering antibody avidity. J Virol. 2016;90(9):4402-4411.

22. Reddy V, Mani RS, Desai A, Ravi V. Correlation of plasma viral loads and presence of Chikungunya IgM antibodies with cytokine/chemokine levels during acute Chikungunya virus infection. J Med Virol. 2014;86(8):1393-1401.

23. Rudolph KE, Lessler J, Moloney RM, Kmush B, Cummings DA. Incubation periods of mosquito-borne viral infections: a systematic review. Am J Trop Med Hyg. 2014;90(5):882-891.

24. Amdekar S, Parashar D, Alagarasu K. Chikungunya virus-induced arthritis: role of host and viral factors in the pathogenesis. Viral Immunol. 2017;30(10):691-702.

25. Wong KZ, Chu JJH. The interplay of viral and host factors in chikungunya virus infection: targets for antiviral strategies. Viruses. 2018;10(6):E294.

26. Tanabe ISB, et al. Cellular and molecular immune response to chikungunya virus infection. Front Cell Infect Microbiol. $2018 ; 8: 345$.

27. Venugopalan A, Ghorpade RP, Chopra A. Cytokines in acute chikungunya. PLoS One. 2014;9(10):e111305.

28. Reddy V, Desai A, Krishna SS, Vasanthapuram R. Molecular mimicry between chikungunya virus and host components: a possible mechanism for the arthritic manifestations. PLoS Negl Trop Dis. 2017;11(1):e0005238.

29. Kam YW, et al. Early neutralizing IgG response to Chikungunya virus in infected patients targets a dominant linear epitope on 
the E2 glycoprotein. EMBO Mol Med. 2012;4(4):330-343.

30. Fox JM, Diamond MS. Immune-mediated protection and pathogenesis of chikungunya virus. J Immunol. 2016;197(11):4210-4218.

31. Chatron P, Pontet F. [Structure an functions of IgG subclasses]. Ann Biol Clin (Paris). 1992;50(8):565-575.

32. Vidarsson G, Dekkers G, Rispens T. IgG subclasses and allotypes: from structure to effector functions. Front Immunol. 2014;5:520.

33. Gunisetty S, et al. Analysis of dengue specific memory B cells, neutralizing antibodies and binding antibodies in healthy adults from India. Int J Infect Dis. 2019;84S:S57-S63.

34. Chandele A, et al. Characterization of human CD8 T cell responses in dengue virus-infected patients from India. $J$ Virol. 2016;90(24):11259-11278

35. Chua CL, Sam IC, Chiam CW, Chan YF. The neutralizing role of IgM during early Chikungunya virus infection. PLoS One. 2017;12(2):e0171989.

36. Raghavendhar BS, Ray P, Ratagiri VH, Sharma BS, Kabra SK, Lodha R. Evaluation of chikungunya virus infection in children from India during 2009-2010: a cross sectional observational study. J Med Virol. 2016;88(6):923-930.

37. B SR, Patel AK, Kabra SK, Lodha R, Ratageri VH, Ray P. Virus load and clinical features during the acute phase of Chikungunya infection in children. PLoS One. 2019;14(2):e0211036. 